\title{
Habla reguladora y control de la memoria: Un esquema teórico de integración de las acciones y operaciones de memorización
}

\author{
MANUEL L. DE LȦ MATA \\ Universidad de Sevilla
}

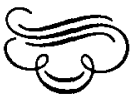

\section{Resumen}

Se propone la integraciōn de varias linneas de investigaciōn de origen diferente: los estudios dentro del marco teórico de niveles de procesamiento, las investigaciones soviéticas sobre memoria y actividad, los trabajos sobre el desarrollo de las estrategias de memoria y los estudios sobre metamemoria y control de la memoria. Para ello se distingue entre tres tipos de procesos: operaciones de codificación y recuperación, acciones simples, ligadas a sus condiciones de aplicación, y acciones generales (planes) que exigen el dominio de otras acciones más simples. Estos tres tipos de procesos se integran de modo jerärquico en un marco teórico general de la memoria. En este marco teórico, el lenguaje (el habla reguladora, más exactamente) juega un papel fundamental como instrumento bäsico de la génesis y control de las acciones de memoria.

Palabras clave: Memoria, desarrollo, niveles de procesamiento, estrategias, acciones, operaciones, control voluntario, habla reguladora.

\section{Regulatory speech and memory actions: a theoretical schema for integrating memory actions and operations}

\section{Abstract}

In this paper a theoretical schema for integrating contributions from different research lines is proposed. These lines are the studies developed within the theory of levels of processing, the soviet investigations about memory and activity, the work on the development of memory strategies and the studies on memory control and metamemory. In order to integrate these, a distinction is made between three kinds of processes: encoding and retrieval operations, simple actions (linked to their conditions of application) and complex, general actions requiring the mastery of simple actions. These processes are hierachically integrated within a general theoretical framework of memory, where language (regulating speech) plays a capital role as the very instrument in the genesis and control of memory actions.

Key words: Memory, development, levels of processing, strategies, actions, operations, voluntary control, regulatory speech.

Dirección de los autores: Universidad de Sevilla. Dto. Psicología Evolutiva y de la Educación, Básica y Metodología. C/ Palos de la Frontera s/n. Aptdo. Corteos 3128. Sevilla 


\section{INTRODUCCION}

En el artículo que precede a éste se ha planteado el desarrollo de la memoria como un proceso de integración jerárquica de acciones simples dentro de otras más complejas, a las que se subordinan las primeras. En este desarrollo la cuestión fundamental sería la adquisición de control por parte del sujeto. Dicho control se adquiere en un proceso que va de fuera a dentro, de las interacciones sociales al sujeto individual. De este modo, el niño adquiere progresivamente un mayor dominio de acciones de memoria cada vez más complejas, que incluyen a otras más simples cuya adquisición fue anterior. Este planteamiento implicaba proponer la noción de Zona de Desarrollo Próximo como marco teórico que permite conceptualizar el desarrollo de las acciones de memoria de forma más completa y realista que otras nociones como la de deficiencia de producción. Todo este planteamiento descansa sobre la integración de las investigaciones soviéticas y occidentales acerca del desarrollo de la memoria, si bien el marco teórico de referencia adoptado es el de la psicología soviética vygotskiana. La integración citada se hacía sobre los conceptos de actividades y procesos de memorización no deliberada (que incluiría las nociones de memoria involuntaria y de aprendizaje incidental) y el de actividades y procesos de memorización deliberada (que incluye los de memoria voluntaria y aprendizaje intencional). A lo largo del artículo se utilizaban como sinónimos los términos estrategia y acción de memoria, si bien conviene aclarar mi preferencia por el término acción, por tener un significado más preciso y por su relación con términos como actividad y operación.

El sentido de este trabajo es avanzar en la línea de una conceptualización global de los procesos de memoria. Para ello se propone un esquema teórico que permita integrar las aportaciones de diferentes líneas de investigación, algunas de las cuales se presentaron en el anterior artículo. Veamos cuales son esas líneas.

- Los trabajos dentro del marco teórico de niveles de procesamiento.

- Las investigaciones de autores soviéticos sobre memoria y actividad.

- Los estudios, de corte evolutivo, sobre todo, acerca de la adquisición y em. pleo de estrategias de memoria.

- Las investigaciones, muy relacionadas con las anteriores, sobre las cuestiones de metamemoria y control de la memoria.

Pese a su origen diverso, estas líneas teóricas y de investigación son integrables en un esquema general de la memoria porque todas ellas comparten un punto esencial: consideran a la memoria como proceso activo, dependiente de la actividad del sujeto en relación con el medio (1). Todo este trabajo va dirigido a exponer el modo en que las líneas citadas pueden encajar en el esquema general de los procesos de memoria. De nuevo la integración se hace desde una perspectiva claramente vygotskiana. Se considera que el desarrollo de la memoria está determinado por la experiencia social del individuo y se otorga al lenguaje el papel fundamental, tanto en el desarrollo, como en el funcionamiento de los procesos de adquisición y recuperación de conocimiento.

En primer lugar se van a exponer los aspectos básicos de las líneas anteriores para, finalmente, defender la forma en que pueden encajar, a mi juicio, en la conceptualización general de los procesos de memoria. 


\section{NIVELES DE PROCESAMIENTO}

El marco teórico de los niveles de procesamiento (sería impropio hablar de la «teoría de niveles de procesamiento) constituye un intento de tratar los problemas de la memoria desde una perspectiva no estructural, como había predominado hasta entonces (ver Atkinson y Shiffrin, 1968, por ejemplo), sino procesual. La idea básica que propusieron Craik y Lockhart (1972) es que «el trazo de memoria es un subproducto del análisis del estimulo y su persistencia constituye una función de la profundidad con la que se analiza el estímulo» (pág. 93). Un análisis profundo, es decir, semántico, del estímulo conduce a una buena retención de éste. Los análisis que quedan en los aspectos más externos y formales del estímulo (por ejemplo, el tipo de letra, mayúscula o minúscula con que está escrita una palabra, el color de un dibujo...) no tienen un efecto tan positivo para la memoria. Para Craik y Lockhart existiría un continuo «que va desde los productos transitorios de los análisis sensoriales a los muy duraderos de las operaciones semántico-asociativas» (pág. 99 de la edición castellana).

Posteriormente, en un trabajo empírico destinado a poner a prueba la concepción de los niveles de procesamiento de Craik y Lockhart, Craik y Tulving (1975) criticaron algunas ideas de aquellos (especialmente la de utilizar el tiempo de reacción como medida de la profundidad de procesamiento y de la retención) y propusieron el concepto de amplitud de la elaboración, básico para explicar los datos discrepantes con lo esperado. Para ellos, la retención del material no depende solamente de la profundidad de análisis, sino que aumenta también en la medida en que sea mayor el grado de elaboración del estímulo. El trazo de memoria puede considerarse un registro de los análisis llevados a cabo sobre el estímulo y su contexto. Cuanto más elaborado sea el contexto de codificación, cuantos más rasgos tenga, más susceptible será de ser empleado para recuperar el estímulo. En palabras de los propios autores:

"La posición que defendemos es la de que el trazo puede considerarse como un registro de las operaciones de codificación del input llevadas a cabo; la función de estas operaciones es analizar y especificar los atributos del estimulo. Pero hay que añadir que el rendimiento de la memoria no puede considerarse simplemente como una función del número de atributos codificados, ya que la naturaleza cualitativa de los atributos tiene una importancia crítica. Una segunda descripción que equivale a la primera es en términos de urasgos compro. bados» durante la codificación. También aqui la comprobación de un mayor número de rasgos (y especialmente de rasgos semánticos) implica un trazo más elaborado (pág. 141 de la trad. castellana).

Por tanto, la retención de la huella de memoria depende de dos factores al mismo tiempo: por un lado de la profundidad a que se analice el estímulo; por otro del grado de elaboración de la codificación, es decir, el número y tipo de rasgos codificados. Hay, pues, un factor puramente cualitativo, la profundidad de análisis, y otro en parte cuantitativo, el grado o la amplitud de la elaboración del código.

Otro concepto, más reciente, dentro del marco general de niveles de procesamiento es el de singularidad o discriminabilidad de la codificación (Jacoby y Craik, 1979). Este concepto subraya el valor de contraste de la información codificada. El significado de un estímulo determinado no es simplemente un atri- 
buto que se codifica o no, sino más bien un conjunto de contrastes que resultan de las distinciones necesarias para interpretar el elemento en el contexto de una tarea determinada. Hay diferencias entre el concepto de singularidad y el de amplitud. La amplitud da idea de un cambio cuantitativo en la codificación, de un añadido de información a la huella. la singularidad, por contra, subraya el valor de contraste de la información. Implica una comparación basada en características comunes.

La idea de contexto está muy presente en este concepto. Una descripción muy singularizada, discriminativa de un item para un conjunto de posibilidades no necesariamente lo es para otro conjunto. Por tanto, no puede especificarse la singularidad de la descripción de un acontecimiento sin tener en cuenta las opciones con las que ha de contrastarse, es decir, el contexto de codificación.

Pero la memorización de una información no depende sólo de como se haya producido la codificación del estímulo. El proceso de recuperación debe considerarse algo determinante también. Esto es lo que hace el concepto de codificación específica (Tulving y Thomson, 1973), desde una perspectiva no plenamente coincidente, aunque próxima a los niveles de procesamiento. El concepto de codificación específica hace referencia a la relación entre los procesos de codificación y recuperación. En su formulación inicial afirma que sólo puede recuperarse aquello que ha sido codificado; la forma en que se puede recuperar depende de la forma en que haya sido codificado. Posteriormente, tras una década de investigaciones relacionadas con el concepto, su formulación se ha matizado. Se afirma ahora que tiene que haber una cierta relación (no especificada) entre las condiciones de codificación y las condiciones de recuperación. El recuerdo de un acontecimiento es función al mismo tiempo de la información almacenada en la memoria acerca del acontecimiento (el trazo) y de la información disponible para el individuo en el momento de la recuperación (la clave) (Tulving, 1979, 1983). No basta con decir que un estímulo determinado se ha codificado de una forma determinada para predecir una fácil o difícil recuperación en cualquier situación. Es necesario que los procedimientos de recuperación reúnan determinadas condiciones para que tengan éxito. Es decir, tiene que darse la relación entre las condiciones de codificación y las condiciones de recuperación antes citadas.

De los conceptos que he introducido en la exposición el de profundidad ha sido el que más cambios ha experimentado. Algunos de estos cambios respecto a la posición inicial son (Craik, 1979):

1) Se supone una mayor flexibilidad en la secuencia de procesamiento. Aunque los niveles de análisis puedan ordenarse en términos lógicos desde superficiales a profundos, la secuencia real que ocurre puede reflejar interacciones y operaciones recursivas entre los varios niveles y tipos de representación. El procesamiento semántico no es un proceso de todo o nada, sino que opera en diferentes grados.

2) La relación entre profundidad de procesamiento y ejecución de memoria es más compleja de lo que se pensaba en principio. No sólo existirían otros factores determinantes de la retención, sino que algunas ocasiones una tarea de procesamiento superficial podría producir mejor retención que el procesamiento semántico, dependiendo de las exigencias de la tarea y de la recuperación. Así, hay autores que defienden la codificación sensorial, mostrando que esta información puede tener una pervivencia mucho ma- 
yor de lo que se podría pensar (Eysenck, 1979; Nelson, 1979; Bransford et al., 1979).

El propio Craik, en suma, reconoce que la generalización previa de que un análisis semántico mínimo es más beneficioso para la memoria que un análisis estructural máximo había sido demasiado rotunda, aunque continúa considerando que la codificación semántica tiene, en general, ventajas para la memoria.

Se puede abandonar en este punto el breve análisis de los conceptos básicos de niveles de procesamiento. Más adelante lo retomaré, para situarlo en el esquema teórico propuesto.

\section{ACCIONES DE MEMORIA}

En el artículo anterior se han presentado las cuestiones fundamentales referidas a este punto. No es necesario repetir aquí los conceptos tratados. Sólo recordar que se incluían las investigaciones y aportaciones teóricas soviéticas y occidentales sobre las actividades y procesos de memorización no deliberada (memoria involuntaria y aprendizaje incidental) y sobre las actividades y procesos de memorización deliberada (memoria voluntaria y aprendizaje intencional). Respecto a la memorización no deliberada, puede recordarse la idea de que estaba determinada por la actividad del sujeto, más que por la existencia o no de intención de memorizar. Esta idea, inicialmente planteada por los psicólogos soviéticos (Smirnov y Zinchenko, 1969; Zinchenko, 1983-84), coincide con lo que defiende una serie de autores occidentales (Brown, 1979; Murphy y Brown, 1975). El trabajo de González y Valle, realizado con niños preescolares y aparecido en esta misma revista (González y Valle, 1984) confirma la misma idea. En cuanto a las actividades y procesos de memorización deliberada, debe tenerse presente el proceso de desarrollo como integración jerárquica de acciones en otras más complejas y la adquisición de control progresivo por parte del sujeto. Para conceptualizar dicho desarrollo se proponía la noción de Zona de Desarrollo Próximo, más apropiada que la de deficiencia de producción y que permite recoger de forma más completa los descubrimientos recientes que dibujan un panorama más optimista de las destrezas de los niños preescolares (Wellman, 1977a, Brown et al., 1983). Igualmente este concepto es más útil para el estudio de la memoria en situaciones naturales. En general, como se afirmaba en el trabajo anterior, la noción de Zona de Desarrollo Próximo se ajusta mejor a una visión continua y compleja del desarrollo de la memoria.

\section{PROCESOS DE CON'TROL}

En el trabajo anterior se resaltaba la importancia que tienen los procesos superiores de control de la memoria. Como se decía, el desarrollo de las estrategias de memoria se considera ligado a la existencia de dichos procesos de control.

Los procesos de control de la memoria incluyen como componente importante el conocimiento de los factores que afectan a la memoria. Durante la década de los setenta y la presente se ha producido una importante cantidad de investigaciones sobre el conocimiento que los individuos tienen de las capacidades de memoria propias y ajenas. Esta investigación se ha centrado en el desarrollo de dos aspectos fundamentales: la conciencia de la necesidad de me- 


\section{4}

morizar (Wellman, Ritter y Flavell, 1975; Kail, 1979; Deloache y Brown, cits. en Brown et al., 1983) y el conocimiento de los diversos factores que afectan a la memoria (Flavell et al., 1970; Kreutzer et al., 1975; Salatas y Flavell, 1976; Flavell, 1977; Flavell y Wellman, 1977). Los primeros trabajos en este campo pintaron un cuadro bastante pesimista de las capacidades de los niños pequeños en este tipo de tareas. Sin embargo, hay que decir que muchos de estos estudios contaron con el inconveniente de estar basados en informes orales de los niños, lo que pone en desventaja a los pequeños. La utilización de métodos que no descansan sobre las destrezas verbales de los niños (Wellman, 1977b; Wellman et al., 1981) ha permitido observar que los niños pequeños ya poseen un cierto conocimiento de los factores que determinan la memoria. Hasta qué edad podrá descenderse en la búsqueda de formas elementales de conocimiento o de precedentes de ese conocimiento es algo que dependerá mucho de la metodología empleada en la investigación.

Centrándonos más específicamente en lo que se ha llamado procesos de control de la memoria hay que decir que algunos de los aspectos más estudiados han sido la capacidad de determinar lo que se ha aprendido (Flavell et al., 1970, Wellman, 1977; Kail, 1979); la de utilizar esa información para el aprendizaje posterior (Masur et al., 1973); o la de juzgar los resultados obtenidos (Moynaham, 1976; Bisanz et al., 1978; Kail, 1979). De nuevo puede decirse que las primeras investigaciones hacían pensar que los niños preescolares o de primer curso no poseían la capacidad de controlar su propia memoria de modo muy eficiente. Las investigaciones del grupo de Brown nos dicen en cambio que los niños preescolares (muy pequeños, incluso) ponen en evidencia capacidades (o al menos, precedentes de capacidades) de control de la memoria, de planificación para el recuerdo, adaptadas a la situación (Brown et al., 1983). Por otra parte, los procesos de control de la memoria continúan desarrollándose más allá de la niñez, hasta la edad adulta.

El estudio de los procesos de control de la memoria (incluyendo el conocimiento de la memoria) evidencia un desarrollo contínuo, que se inicia muy pronto y llega hasta la etapa adulta, por el que el individuo va adquiriendo un dominio progresivo de destrezas que le permiten enfrentarse a tareas cada vez más difíciles y en situaciones cada vez más exigentes, empleando estrategias finas y sofisticadas cuya aplicación puede regular de forma adaptada y flexible. Existe una clara continuidad entre el desarrollo de lo que se ha llamado estrategias de memoria y lo que aquí se ha denominado destrezas de control de la memoria. En ambos casos, la clave es el control de las acciones de memoria, el proceso de inclusión, subordinación y jerarquización de acciones y operaciones, de las que el individuo va adquiriendo un dominio creciente. Este proceso general de jerarquización culmina con la constitución del sistema ejecutivo de control (Brown, 1978), ya citado en el anterior artículo, capaz de elaborar y poner en juego planes generales de memoria. En todo el proceso, el lenguaje juega un papel primordial como instrumento que permite la elaboración y utilización de los planes del sistema de control (Lefebvre-Pinard, 1982).

\section{HABLA Y CONTROL DE LA MEMORIA}

En este apartado voy a examinar brevemente la intervención del lenguaje en la regulación de la memoria. Interesa el aspecto de actividad del lenguaje, no 
su vertiente de sistema de signos abstractos. Es preferible utilizar el término habla, que tiene un significado de actividad funcional más definido. Para estudiar la intervención del habla en la regulación de la memoria es necesario detenerse en las características del habla reguladora, especialmente la predicatividad del habla interiorizada, que Wertsch (1979) interpreta desde la noción de conciencia y los conceptos de «lo nuevo» y «lo conocido (Chafe, 1976). Hecho esto, entraré en el análisis de la intervención del habla en la regulación de la memoria, desde una perspectiva coincidente con la Teoría de la Modulación de la Actividad (Ramírez, en prensa), que hace referencia a los factores implicados en la regulación verbal.

El habla interiorizada de Vygotski (1977) es el estadio final del proceso de interiorización y de pérdida del aspecto vocal que sigue el lenguaje. La característica fundamental de este habla es la tendencia a la predicación, que constituye su forma sintáctica básica.

Desde este momento utilizaré el término habla privada, que incluye al habla interiorizada y al habla egocéntrica (precedente genético de ésta que comparte, aunque de forma menos acentuada, sus características funcionales). Este término, pese al problema de la fuerte connotación de voluntariedad que tiene, y que excluiría de modo explícito el habla usada involuntariamente, ha sido utilizado ampliamente, junto al de «habla para uno mismo», para designar al tipo de habla no comunicativa presumiblemente autorreguladora (Zivin, 1979).

Se ha afirmado que el rasgo principal del habla egocéntrica e interiorizada (el habla privada) es su carácter predicativo. Para Vygotski, el habla predicativa carece de sujeto psicológico, sólo posee predicado. El sujeto y el predicado psicológicos no coinciden con el sujeto y el predicado gramaticales. Vygotski emplea un ejemplo ilustrativo para explicar el concepto de predicado psicológico.

- En una parada de autobús hay varias personas esperándolo. Si en un momento determinado, tras un rato de espera, una de estas personas dice la frase «Ya viene», los demás entenderán que se refiere al autobús. En otra situación la frase no sería comprendida, pero en ésta sí, puesto que todos comparten el contexto (la espera y el deseo de que llegue el autobús). En el habla privada ocurre algo parecido. Cuando un niño, por ejemplo, utiliza el habla egocéntrica para resolver un problema, él conoce el «contexto» de la emisión verbal, la situación en que se produce (la de resolución del problema) y, consecuentemente, sólo verbaliza lo que no viene dado en el contèxto. El sujeto psicológico es justamente aquello que no queda definido por el contexto, el «tema» de la comunicación, como afirma Luria (1980). El predicado psicológico es lo que se dice acerca del sujeto psicológico, lo que la emisión verbal introduce que no estaba definido en el contexto. En lingüística se denomina «rema» (Luria, 1980).

Wertsch (1979) interpreta los conceptos de sujeto y predicado psicológico desde la noción de conciencia. La distinción sería semejante a la que hace Chafe (1976) entre información «dada» o «conocida» («given») e información «nueva» («new»). La información dada o conocida es la que el hablante supone que está en la conciencia del oyente en el momento de la emisión verbal. La información nueva es la que el hablante considera que está introduciendo por medio del mensaje que emite. La parte de la emisión verbal que lleva la información conocida se caracteriza, a juicio de Chafe, por recibir baja entonación y su tendencia a la pronominalización. En cambio, la parte que expresa la información nueva recibe mayor énfasis. 
Tanto Vygotski como Chafe dan gran importancia a la idea de conciencia a la hora de distinguir entre el sujeto y el predicado psicológico o entre la información conocida y nueva. Sin embargo, hay algo que diferencia a los dos enfoques. El análisis de Chafe implica un intercambio comunicativo en el que participan al menos dos personas, mientras que la distinción que hace Vygotski se refiere a situaciones que involucran a una sola persona. Por tanto, la distinción sujeto-predicado psicológico no puede hacerse sobre la base de lo que una persona introduce en la conciencia de otra. Wertsch propone que se defina lo conocido y lo nuevo en el habla comunicativa sobre la base de la acción del individuo más que sobre la de la interacción con otra persona. De este modo la información conocida sería el conocimiento que está en la conciencia del hablante en el momento de la emisión verbal reguladora (en el momento en que está realizando la acción). La información nueva, por su parte, sería lo que está siendo introducido en la conciencia del hablante como resultado de la acción que está ejecutando.

La organización del habla privada, lo que es nuevo y lo que es conocido, no está determinada por los factores contextuales ni por los objetos en sí mismos. Lo determinante es cómo encajan los objetos en la acción dirigida hacia una meta. Considerar los objetos en sí mismos, aparte de la acción en que se insertan, no puede decirnos, en opinión de Wertsch, qué aspectos de la situación se relacionan con la información conocida. Sería posible utilizar los mismos objetos en diferentes situaciones, de manera que cambiara la organización de la información nueva y conocida, y el habla privada lo reflejaría. Esto podría hacerse cambiando la meta de la acción, con lo que cambiaría la acción misma, definida por la meta.

El papel que el habla privada cumple es el de reflejar las acciones del individuo. En este contexto, el término acción debe entenderse como conducta dirigida a un fin. Las acciones pueden ser acciones externas (motoras) o acciones internas, realizadas en el plano del pensamiento. El papel del lenguaje en la regulación de las acciones ha sido estudiado en Occidente respecto a las acciones motoras, sobre todo. Sin embargo, este papel regulador no se agota para la psicología soviética en las acciones externas motoras, sino que se extiende también a las acciones internas. Entre ellas lo que aquí me interesa son las que permiten al individuo adquirir conocimientos, más o menos generales acerca de su medio. Es decir, la participación del lenguaje en la regulación y control de las acciones de memoria. Analicemos cómo puede hacerlo.

Etiquetar o rotular un estímulo (decir su nombre) es una acción, una estrategia simple de ayuda a la memoria. Tiene un efecto positivo sobre la memoria de los niños pequeños, sobre todo en tareas de recuerdo serial (Hagen y Kingsley, 1968). Sin embargo, para los niños mayores el simple etiquetado puede tener efectos negativos al impedir que empleen otro tipo de estrategia más eficaz.

Otra acción de memoria verbal que emplean los individuos en una gran cantidad de situaciones y que ha sido muy estudiada es el repaso (Flavell et al., 1966; Keeney et al., 1967; Kingsley y Hagen, 1969; Ferguson y Bray, 1976; Kennedy y Miller, 1976; Gelabert et al., 1980). El repaso verbal aparece alrededor de los primeros años escolares. Una vez que ha comenzado, el repaso espontáneo cambia con la edad, desde la simple repetición de los estímulos tal como fueron presentados, hasta una repetición organizada de los items (Ornstein et al., 1975). La acción de repasar, en principio muy simple, se va haciendo más compleja en la medida en que incluye otras acciones simples en su seno (que 
se convierten de este modo en operaciones). Esta progresión evolutiva lleva aparejado el que una instrucción de verbalizar un estímulo particular pueda facilitar la ejecución (en los niños que lo hacen espontáneamente), o dificultarla (si esta verbalización evita el uso de otra verbalización más efectiva) (Fuson, 1979). La acción de repasar como ayuda a la memoria puede, por otra parte, tener carácter abierto o encubierto.

Otros ejemplos de acción verbal más sofisticada son la elaboración verbal y el uso de términos categoriales para agrupar los items de recuerdo. Incluso los preescolares son capaces de elaborar verbalmente estímulos con efecto positivo para el aprendizaje de pares asociados (Pressley, 1982). El empleo de términos categoriales es una estrategia de considerable valor. Cuando el término verbal se suministra al sujeto en el momento de la presentación del material, las diferencias evolutivas se reducen y los niños pequeños alcanzan resultados, tanto en número de items como en la organización de esos items, más próximos a los de los niños de diez-once años (Moely et al., 1969; Kee y Bell, 1981). En los niños preescolares estrategias de elaboración verbal como construir una frase que ponga en relacion dos items en función de sus relaciones concretas parece más eficaz que el empleo de un término categorial que ponga en relación a ambos elementos. No obstante, esta conclusión debe matizarse según la tarea en cuestión (Moely, 1977; Bjorklund y Zeman, 1982; Bjorklund y de Marchena, 1984).

La utilización de un término categorial no sólo es eficaz en la adquisición, sino que ha probado su valor igualmente como estrategia de recuperación (Halperin, 1974; Kobasigawa, 1974; Kee y Bell, 1981). No hay acuerdo, no obstante, sobre en qué medida la instrucción de su empleo, realizada por el experimentador, reduce las diferencias evolutivas en el número de elementos recordados (Kobasigawa, 1974; Kee y Bell, 1981).

Cuando el sujeto nombra el estímulo en la tarea de etiquetado o repaso, nombra la categoría en la adquisición o en la recuperación, o construye una frase que pone en relación dos estímulos está diciendo algo nuevo del material en el sentido de Wertsch (1979). Se está predicando algo de los estímulos, los cuales son, en este caso, lo conocido, lo ya dado. Hay, por tanto, una intervención del habla reguladora, abierta o encubierta, que pone en el foco de la conciencia lo nuevo, modificando las acciones internas, es decir, el conocimiento del sujeto. Este conocimiento puede ser de índole particular y concreta, comó el orden en el que el experimentador señaló varios dibujos en una tarea de recuerdo serial, o de naturaleza más general y conceptual, como la pertenencia de un determinado estímulo a una clase de inclusión (3).

Pero no sólo puede decirse que el lenguaje interviene decisivamente como componente de las estrategias de memoria. Desde la posición que defiendo aquí, el lenguaje es el instrumento principal por el que se realiza la autorregulación de la memoria. El lenguaje permite la construcción de planes de memoria. En estos planes, la función del lenguaje consiste en: (Ramírez, 1984)

- Desarrollar un conocimiento diferenciado acerca de los diversos factores que pueden afectar al curso de una acción cognitiva como la memorización.

- Formular los objetivos de la acción, lo que ayuda a fijarlos.

- Generar y controlar planes de autoinstrucciones. Incluso los más pequeños pueden dirigir su actividad de memoria usando planes apropiados de autoinstrucciones.

- Evaluar el cumplimiento de los objetivos propuestos. 
En los planes de memoria el habla reguladora interviene del modo que determinan sus características funcionales. Introduce en cada momento lo que es relevante para la ejecución del plan (lo «nuevo» de Wertsch) y la consecución de los objetivos establecidos.

\section{CONCLUSIONES}

Hasta aquí he hecho una revisión de las nociones básicas de varias líneas de investigación sobre la memoria. Es el momento de presentar la forma en que pueden integrarse en un esquema teórico general de los procesos de memoria. La idea básica es que, aunque existen diferencias de índole teórica entre unas líneas y otras, es posible integrarlas, dado que se sitúan a diferente nivel de análisis de los procesos de memoria. Se centran en el estudio de procesos de diferente grado de generalidad, de modo que unos enfoques y otros pueden resultar complementarios.

Las investigaciones realizadas dentro del marco de niveles de procesamiento, pese a enmarcarse en una línea teórica surgida en el contexto general de la llamada «psicología del procesamiento de la información» (y pueden hallarse huellas de este «paradigma», por ejemplo, en la secuencia fija inicial de análisis de lo estructural a lo semántico), tienen el gran acierto de centrarse en el aspecto activo de la memoria, en el «qué hace» el individuo con la información. Este planteamiento permite hacer una lectura que conciba los análisis realizados sobre la información como operaciones (o. acciones simples, en ocasiones) insertas en la actividad del sujeto, es decir, en el curso de la interacción entre el individuo y su medio que explica el desarrollo de los procesos psicológicos. Los estudios de niveles de procesamiento se han acercado a los procesos de adquisición (y recuperación) de conocimiento desde un punto de vista «molecular», de detalle. Su foco de atención han sido procesos bastante simples, moleculares. Conceptos como profundidad, amplitud, singularidad y codificación específica tratan de definir el tipo de análisis específicos que los individuos efectúan sobre los estímulos, es decir, los mecanismos por los que tiene lugar el análisis de la información. Desde una terminología soviética podría decirse que se han centrado en el estudio de lo que, en el contexto de las estrategias de memoria serán operaciones de codificación y tecuperación de información, componentes de acciones (estrategias) de memoria.

Las investigaciones soviéticas y occidentales acerca de la memoria voluntaria y el uso de estrategias se han dedicado al estudio de procesos más generales que los que han constituido el objeto de estudio de los teóricos de niveles de procesamiento. Sin excluir por completo el análisis de procesos más simples, lo que ha atraído su interés ha sido el descubrir cómo los sujetos humanos van adquiriendo procedimientos activos de memorización y cómo esos procedimientos van siendo dominados de forma espontánea y cada vez más completa y adaptada (por ejemplo, la evolución que hace que una estrategia, que en cierto momento solo podía aplicarse cuando el adulto guiaba su empleo mediante instrucciones, termina siendo aplicada por el niño sin ayuda externa). Lo que se ha estudiado, pues, son los procesos de alcance más «molars, más general que los anteriores. Estos procesos más generales incluyen procesos más simples (operaciones) (4), que quedan insertos de modo subordinado en el proceso más general. Desde el punto de vista soviético lo que se estudia son acciones de me- 
moria, y especialmente la forma en que esas acciones se originan y se van perfeccionando.

\section{FIGURA 1}

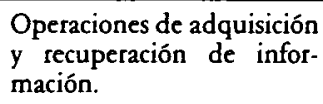

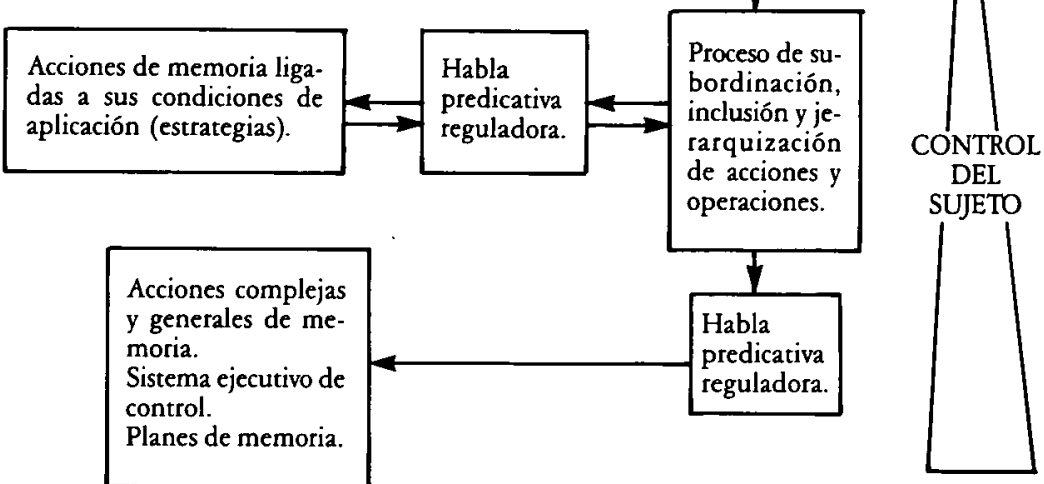

- El cuadro 1 presenta el esquema teórico de integración jerárquica de los procesos de memoria que se ha propuesto. En la parte superior estān los procesos más simples, las operaciones de adquisición y recuperación (estudiadas por los autores de niveles de procesamiento). A continuación aparecen las acciones de memoria de aplicación relativamente restringida y vinculadas a sus condiciones de aplicación. Este tipo de procesos incluye la mayoría de las estrategias de memoria estudiadas por autores occidentales y soviéticos (repaso, categorización, elaboración verbal, etc.) En el nivel superior (en cuanto a generalidad, aunque en la parte baja del cuadro) se encuentran los llamados pro. cesos de control, que dirigen el uso de estrategias y culminan en el sistema ejecutivo de control de la memoria, encargado de elaborar y poner en funcionamiento los planes de memoria. Los tres tipos de procesos se relacionan entre si de modo jerárquico. Desde este planteamiento, el desarrollo de la memoria es un proceso general de inclusión, subordinación y jerarquización de acciones $y$ operaciones en el que los elementos más simples van quedando incluidos como constituyentes de otros elementos de superior complejidad. En todo el proceso de desamollo y en el funcionamiento de los distintos elementos, el babla reguladora tiene un papel primordial; especialmente interviene en gran número de estrategias, de naturaleza total o parcialmente verbal y, sobre todo, en los componentes superiores del modelo (planes de memoria), de caräcter esencialmente verbal. Todo el cuadro, por último, está articulado en torno a la idea de control, por parte del sujeto, de los diferentes procesos. Este control es mayor cuanto más completo es el proceso considerado, de modo que el máximo grado se encuentra en las acciones generales y complejas de memoria, los planes de memoria.

El modelo propuesto tiene una doble dimensión: por una parte presenta simultáneamente todos los elementos constituyentes, y por otra, apunta un importante sentido evolutivo, de construcción de procesos en el tiempo. Esta doble dimensiōn no surge de una confusión de los aspectos diacrónicos y sincrónico de la memoria, sino del reconocimiento de que lo evolutivo tiene una importancia esencial en todo proceso psicológico y está presente incluso en el funcionamiento del sistema (que, por otra parte, nunca alcanza un estadio final, sino que está en continua transformación, en continuo desarrollo).

Por último, el estudio de la metamemoria y el control de la memoria se han centrado en los procesos más complejos y generales, que incluyen a todos los anteriores. Las destrezas de control exigen decidir acerca del uso de una estrategia, adaptándolo a la tarea en cuestión (el objetivo de la acción general), a las condiciones específicas en que dicha estrategia debe aplicarse, así como dirigir el empleo de la estrategia y decidir su efectividad de acuerdo con el objetivo preestablecido. Se trata, por tanto, de acciones muy generales y complejas (planes) que incluyen otras acciones más simples. Tal como señalan Brown y Deloa- 
che (1978), los procesos de control implican combinar una estrategia general, que supone conciencia y realización de planes, con otras específicas a la tarea. Manteniendo la coherencia de la propuesta con el planteamiento de la psicología soviética hay que señalar que en el contexto de los planes generales de memoria, las acciones más simples (estrategias) que se insertan en el plan, deben caracterizarse como operaciones, es decir, medios de realización de la acción principal (plan de memoria).

La figura 1 resume el esquema teórico propuesto. En primer lugar aparecen los procesos simples (operaciones) de adquisición y recuperación caracterizados por los conceptos de niveles de procesamiento. A continuación, en un grado de generalidad superior, las acciones de memoria (estrategias). Se han considerado simples en la medida en que están ligadas al tipo de material específico al que se aplican y para distinguirlas de las acciones generales y «descontextualizadas» que constituyen el dominio de las destrezas de control de la memoria. Hay una idea importante que puede dar coherencia al conjunto; que es la de control. Todo el proceso de desarrollo de la memoria es un proceso de adquisición de control de las propias capacidades de memoria. Al mismo tiempo, en la medida en que tratamos con procesos más generales y «molares», la capacidad de control que su empleo exige es mayor también. La utilización de acciones generales (planes de memoria) lleva consigo un grado de control mayor que el empleo de estrategias simples. Del mismo modo, las estrategias simples exigen nuestro control por el sujeto. En el caso de las operaciones estudiadas por los teóricos de niveles de procesamiento lo importante es el estudio de la operación de análisis en sí misma, no el hecho de que el control corresponda al sujeto o a la tarea (la tarea de orientación).

El último elemento importante de este cuadro es el lenguaje, cuya intervención ya se ha analizado. Sólo quiero resaltar que la intervención del lenguaje está estrechamente vinculada a la idea de control. En la medida en que tratamos con procesos que exigen un mayor control al sujeto, la intervención del lenguaje es más esencial. El lenguaje está presente en algunas de las operacio-

\section{FIGURA 2}

INFORMACION: registrada en la interacción S-MA

puede ser manipulada o transformada por diferentes
PROCESOS:

Sistema de procesamiento de información

(Tomado de Lawson, 1980, pág. 147). El cuadro muestra el modo en que se articulan los diferentes elementos del sistema de procesamiento de información: procesos, estrategias y metacognición. El esquema es válido no sólo para la memoria, sino también para otras funciones psicológicas (resolución de problemas, razonamiento, etc.). Los procesos constituyen el nivel más elemental. A continuación, por orden de generalidad, irian las estrategias, pretrequisitos lógicos de la metacognición, puesto que deben estar presentes antes de que el sujeto pueda tomar decisiones acerca de su uso. La metacognición constituye el nivel superior del sistema. 
nes de codificación y recuperación (por ejemplo, los análisis semánticos tienen un importante componente verbal) y en muchas estrategias de memoria, como se ha visto. Pero su intervención es decisiva en los procesos de control. Tal como se ha dicho, el lenguaje es el instrumento básico de autorregulación de la conducta, permitiendo la construcción y el funcionamiento de planes de memoria, de carácter verbal.

El esquema teórico propuesto tiene puntos en común con la concepción general del sistema de procesamiento de la información de Lawson (1980).

En el cuadro se observa que la información registrada en la interacción con el medio puede considerarse a diferentes niveles. En el nivel inferior están los procesos, que suponen un análisis inicial de los items, registro de atributos y establecimiento de la representación de los items de la lista. A un nivel superior están las estrategias, de acuerdo con las cuales pueden utilizarse los elementos del nivel de procesos. Las estrategias pueden ser de adquisición (vincular items por pares, repaso, generar imágenes interactivas) y de recuperación (buscar la lista usando un asociado o imagen como clave...). Por último, el nivel superior es el metacognitivo, que incluye la toma de una serie de decisiones: selección de estrategias, control de la ejecución de éstas, etc. Los dos niveles inferiores, de procesos y de estrategias son prerrequisitos lógicos del nivel metacognitivo, puesto que las estrategias ( $y$, por tanto, los procesos) deben estar presentes antes de que el sujeto pueda tomar decisiones acerca de su empleo.

Pese a la mayor formalización de la propuesta de Lawson, parece interesante la que he presentado anteriormente ya que introduce los conceptos de acción y operación, más precisos que los de proceso y estrategia y que dan una idea más clara del modo en que se relacionan jerárquicamente los elementos de un nivel de análisis con los de otros niveles más generales o más específicos. Por otra parte, la inclusión del lenguaje como instrumento básico de los procesos implicados, especialmente los que exigen mayor grado de control, marca tambiến una diferencia con el modelo de Lawson. La inclusión del lenguaje, además, remite claramente a interacción social como base de la formación de las acciones de memoria. De todos modos, el esquema propuesto no deja de ser un primer esbozo de lo que sería un modelo general de los procesos activos de memoria, que debe desarrollarse en diferentes direcciones:

- Aclarar los mecanismos de inclusión jerárquica por los que los procesos más simples y particulares se van insertando como operaciones de acciones de alcance más general, tanto de índole estratégica como de ámbito superior, cuyo control va asumiendo el sujeto.

- Cuáles son los mecanismos por lo que se produce la regulación verbal de las acciones (simples y complejas) de memoria. En este sentido, es necesario vincular las experiencias de interacción social con la cualidad del habla reguladora y ésta con los procesos de memoria del individuo.

- Puesto que la posición general que defiendo es que los procesos psicológicos están estrechamente vinculados a las condiciones de vida de los individuos, es necesario determinar con mayor claridad la influencia de los aspectos culturales, y entre ellos algunos tan importantes como la escolarización, el medio (rural y urbano), la actividad laboral, etc. en la génesis y funcionamiento de las acciones de memoria (especialmente los procesos de control de mayor gene- 
ralidad), aclarando también qué aspectos de estos procesos se relacionan con algunas de las condiciones de vida de los individuos de nuestra cultura y otras culturas.

\section{Notas}

${ }^{1}$ En este punto no hay que entender el término actividad en el sentido preciso que le dan los psicólogos soviéticos, sino en un sentido más laxo, como sinónimo de actuación del sujeto.

2 Aquí sí aparece, en cambio, el término actividad, junto al de acción, en la acepción precisa de la psicología soviética de línea vigotskiana. Remito al artículo anterior, en el que se explican los términos actividad, acción y operación.

${ }^{3}$ Se está hablando de los tipos de información propios de la memoria espisódica, desde una perspectiva clásica. Es preferible, a mi juicio, adoptar el punto de vista de Craik y Jacoby (1979) que defienden una jerarquización de la información desde conocimientos muy ligados a lo concreto y al contexto en que fueron adquiridos, hasta otros de tipo general y abstracto, independientes de lo contextual, pasando por conocimientos intermedios, en cuanto a su grado de descontextualización, entre la base, plenamente concreta, de la jerarquía, y los conocimientos más abstractos. Esta conceptualización, que permite responder a la cuestión del origen de lo ssemánticos desde lo «episódico», parece más realista que la que distingue entre información procedente del sistema semántico e información procedente del sistema episódico.

${ }_{4}$ Podría ponerse el ejemplo de una estrategia de elaboración, por la que un sujeto inventa una frase que pone en relación dos palabras o dos dibujos. Desde la perspectiva de estudio de la memoria adoptada por los autores de niveles de procesamiento, lo interesante sería el tipo de análisis, superficial o profundo, efectuado para relacionar los dos estímulos. Es decir, si se relacionan sobre la base de semejanzas fonéticas (en el caso de dos palabras) o sobre la base de la pertenencia a la misma categoría. Los estudiosos de estrategias, en cambio, se interesarían más bien por la capacidad del individuo para emplear la estrategia de modo espontáneo o con ayuda, teniendo éxito o no teniéndolo, y por la forma en que se desarrolla la estrategia.

\section{Referencias}

AtKINSON, R.C. y SHIFFrin, R.M. (1968). Human memory: a proposed system and its control. En K.W. Spence y J.T. Spence (Eds.): The psychology of learning and motivation (Vol. II) Nueva York. Academic Press (Trad. castellana en M.V. Sebastian (Comp.): Lecturas de psicología de la memoria. Madrid: Alianza, 1983).

BISANZ, G.L; VESONDER, G.T. y VOSS, J.F. (1978). Knowledge of one's own responding and the relation of such knowledge to learning: A developmental study. Joumal of Experimental Cbild Psycho$\log y, 25$ 116-128.

BJORKUND. D. y de MARCHENA, M.R. (1984). Developmental shifts in the basis of organization in memory: the role of associative versus categorical relatedness in children's free recall. Child Development, 55, 3, 952-962.

BJORKUNND, D. y ZEMAN, B. (1982). Children's organization and metamemory awareness in their recall of familiar information. Child Development, 53, 799-810.

BRANSFORD, J.; FRANKS. J.; MORRIS, C.D. y STEIN. B.S. (1979). Some general constraints on learning and memory research. En L.S. Cermak y F.I.M. Craik (Eds.): Levels of processing in buman memory. Hillsdale: L.E.A.

BROWN, A.L. (1978). Knowing when, where and how to remember: a problem of metacognition. En R. Glaser (Ed.) Advances in instructional psychology (Vol. 1). Hillsdale: L.E.A.

Brown, A.L. (1979). Theories of memory and the problem of activity, growth and knowledge. En L.S. Cermak y F.I.M. Craik (Eds.): Levels of processing in human memory. Hillsdale: L.E.A.

Brown, A.L.; BransFord, J.D.; FerRARa. R.A. y Campione, U.C.: Learning, remembering and understanding. En J.H. Flavell y E. Markman (Eds.): Cognitive development. Nueva York: Wiley.

Brown, A.L. y DELOACHE, J.S. (1978). Skills, plans and self-regulation. En R.S. Siegler (Ed.): Children's thinking. What develops? Hillsdale: L.E.A.

CraIK. F.I.M. CRAIK (1979). Levels of processing: overview and closing comments. En L.S. Cermak y F.I.M. Craik (Eds.): Levels of processing in human memory. Hillsdale: L.E.A.

CRAIK, F.I.M. y LOCKHART, R.S. (1972). Levels of processing: a framework for memory research. Joumal of Verbal Learning and Verbal Behavior, 11 (trad. castellana en Estudios de Psicologia, 2, 1980, 110-146.

CRAIK, F.I.M. y JACOBY, L.L. (1979). Elaboration and distinctiveness in episodic memory. En L.G. Nilsson (Ed.): Perspectives on memory research. Hillsdale: L.E.A.

CRAIK, F.I.M. y TuIVING, E. (1975). Depth of processing and the retention of words in episodic me- 
mory. Joumal of Experimental Psychology: General, 104, 268-294 (trad. castellana en Estudios de Psicología, 1980, 2, 110-146).

CHAFE, W.L. (1976). Giveness, contrastiveness, defineteness. Subject, topics and point of view. En C.N. Li (Ed.): Subject and topic. Nueva York: Academic Press.

DRAKER, F. y HAGEN.J.W. (1969). Developmental trends in the processing of task relevant and task irrelevant information. Child Development, 40, 371-382.

EYSENCK. M.W. (1979). Depth, elaboration and distinctiveness. En L.S. Cermak y F.I.M. Craik (Eds.): Levels of processing in buman memory. Hillsdale: L.E.A.

FERGUSON, R.P. y BRAY, N.W. (1976). Component processes of an overt rehearsal strategy in young children. Journal of Experimental Child Psychology, 47, 556-559.

FLAVEI, J.H. (1970). Developmental studies of mediated memory. En H.W. Reese y L.P. Lipsitt (Eds.): Advances in child development and behavior (Vol. 5). Nueva York: Academic Press.

Flaveu. J.H. (1977). Cognitive development. Englewood Cliffs. Prentice-Hall (trad castellana en Aprendizaje-Visor, Madrid, 1984).

FlaveIL, J.H.; BeACH. D.H. y ChinSKY. T.M. (1966). Spontaneous verbal rehearsal in a memory task as a function of age. Child Development, 37, 283-299.

FLAVEL. J.H.; FrIEDRICHS, A.G. y HOYT, J.D. (1970). Developmental changes in memorization processes. Cognitive Psychology, 1, 324-340 (trad. castellana en J. Delval (Comp.): Lecturas de psicología del niño (Vol. 2). Madrid: Alianza, 1978).

Flaveli.J.H. y Wellman. H.M. (1977) Metamemory. En R.V. Kail y J.W. Hagen (Eds.): Perspectives on the development of memory and cognition. Hillsdale: L.E.A.

FUSON, K. (1979). The development of self-regulating aspects of speech: a review. En G. Zivin (Ed.): The development of self-regulation through private spech. Nueva York: Wiley.

GelaberT. T.; TORGESSEN. J.; DiCE. C. y MURPHY, H. (1980). The effects of situational variables on the use of rehearsal by first-grade children. Child Development, 51, 3, 902-905.

GONZALEZ. M.A. y VAUE. F. (1984). Memoria incidental e intencional en los niños preescolares. Infancia y Aprendizaje, 27-28, 223-235.

HAGEN, J.W. (1967). The effect of distraction on selective attention. Child Development, 38 , 371-382.

HAGEN, J.W. y KINGSLEY, P.R. (1968). Labeling effects in short-term memory. Child Development, $39,113-121$.

HaGen, J.W. y Stanovich, K.G. (1977). Memory: strategies of acquisition. En R.V. Kail y J.W. Hagen (Eds.): Perspectives on the development of memory and cognition. Hillsdale: L.E.A.

HAIPERIN. M.S. (1974). Developmental changes in the recall and recognition of categorized words lists. Child Development, 45, 144-151.

ISTOMINA, Z.M. (1975). The development of voluntary memory in preschool age children. Soviet Psychology, 2, 5-65.

JACOBY, L.L. y CRAIK, F.I.M. (1977). Effects of elaboration of processing in encoding and retrieval: Trace distinctiveness and reconvery of initial context. En L.S. Cermak y F.I.M. Craik (Eds.): Levels of processing in human memory. Hillsdale: L.E.A. (Trad. castellana en M.V. Sebastian (Comp.): Lecturas de psicología de la memoria. Madrid: Alianza, 1983).

KAIL, R.V. (1979). The development of memory in children. San Francisco: Freeman \& Co. (Trad. castellana en Madrid: Siglo XXI, 1984).

KEE, D. y BELL. T.S. (1981). The development of organizational strategies in the storage and retrieval of categorial items in free-recall learnings. Child Development, 52, 4, 1163-1171.

KeENEY, T.J.; CANNizo, S.R. y Flaveul. J.H. (1967). Spontaneous and induced verbal rehearsal en a recall task Child Development, 38, 953-966.

KENNEDY, B.A. y MILER. D.J. (1976). Persistent use of verbal rehearsal as a function of information about its value. Child Development, 47, 566-569.

KINGSLEY. P.R. y HAGEN. J.W. (1969). Induced versus spontaneous rehearsal in short-term memory in nursery school children. Developmental Psychology, 1, 40-46.

Kobasigawa, A. (1977). Retrieval strategies in the development of memory. En R.V. Kail y J.W. Hagen (Eds.): Perspectives on the development of memory and cognition. Hillsdale: L.E.A.

KREUTZER, M.A.; LEONARD. C. y FlaveU, J.H. (1975). An interview study of children's knowledge about memory. Monographs of the Society for Research in Child Development, 40. 1. 1.58.

LAwson, M.J.: Metamemory: making decisions about strategies. En J.R. Kirby y J.B. Bigss (Eds.): Cognition, development and instruction. Nueva York: Academic Press. 1980.

LEFEBVRE.PINARD. M. (1983). Understanding and auto-control of cognitive functions: implications for the relationship between congnition and behavior. International Jomal of Behavior Develop. ment, 6, 15-35.

LURIA. A.R. (1980). Conciencia y lenguaje. Madrid: Pablo del Río.

MASUR, E.F; McINTYRE. C.W. y FLAVEII. J.H. (1973). Developmental changes in apporioment of study time among items in a multitrial free recall task. Joumal of Experimental Child Psychology. 15. 237-246.

MeACHAM. J.A. (1972). The development of memory abilities in the individual and sociery. $\mathrm{Hu}$. man Development, 15, 205-228.

MeACHAM, J.A. (1977). Soviet investigations of memory development. En R.V. Kail y J.W. Hagen (Eds.): Perpectives on the development of memory and cognition. Hillsdale: L.E.A.

MOEIY. B.E. (1977). Organizational factors in the development of memory. En R.V. Kail y J.W. Ha- 
gen (Eds.): Perspectives on the development of memory and cognition. Hillsdale: L.E.A.

MOELY, B.E.; OLSON, F.A.; HaLWES, T.G. y FlaveIL. J.H. (1969). Production deficiency in young children clustered recall. Developmental Psychbology, 1, 26-34.

Mочмлнлм, G.D. (1976). The development of the ability to asses free recall performance. Joumal of Experimental Child Psychology, 21, 94-97.

MURPHY, M.D. y BROWN, A.L. (1975). Incidental learning in preschool children as a function of level of cognitive analysis. Joumal of Experimental Child Psychology, 19, 509-523.

NELSON, D.L. (1977). Remembering pictures and words: appearance, significance and name. En L.S. Cermak y F.I.M. Craik (eds.): Levels of processing in buman memory. Hillsdale: L.E.A.

ORNSTEIN, P.A.; NAUS, M.J. y LIBERTY, C (1975). Reshearsal and organizational processes in children's memory. Child Development, 46, 818-830.

Pressley, M. (1982). Elaboration and memory development. Child Development, 53, 2, 296-309.

RAMirEZ, J.D. (1984). El lenguaje como instrumento regulador de la conducta. En M. Carretero, A. Marchesi y J. Palacios: Psicología Evolutiva (Vol. 2). Madrid: Alianza.

RAmireZ, J.D. Desarrollo del lenguaje y control de las acciones. En torno a la regulación verbal. Infancia y Aprendizaje. (en prensa)

SABO. H. y HAGEN, J.W. (1973). Behavioral and metamnemonic indicators of strategic behaviors under remembering instructions in first grade. Child Development, 47, 81-89.

SMIRNOV, A.A. y ZINCHENKO. P.I. (1969). Problems in the psychology of memory. En M. Cole e I. Maltzman (Eds.): A handbook of contemporary Soviet psychology. Nueva York: Basis Books.

Turving, E. (1979). Relation between enconding specificity and levels of processing. En L.S. Cermak y F.I.M. Craik (Eds.): Levels of processing in buman memory. Hillsdale: L.E.A.

Tulving, E. (1983). Elements of episodic memory. Oxford: Clarendon Press.

Tulving, E. y Thomson, D.M. (1973). Enconding specificity and retrieval processes in episodic memory. Psychological Review, 80, 352-373. (Trad. castellana en M.V. Sebastian (Comp.): Lecturas de psicología de la memoria. Madrid: Alianza.

VYGotsKI, L.S. (1977). Pensamiento y leng̨uaje. Buenos Aires: La Pléyade.

Weuman. H.M. (1977a). The early development of intentional memory behavior. Human Development, 20, 86-101.

Welman. H.M. (1977a). Tip of the tonge and feeling of knowing experiences: a developmental study of memory-monitoring. Child Development, 48, 13-21.

Wellman, H.M.; Coluns. J. y Gleberman. J. (1981). Understanding the combination of memory variables: developing concepts of memory limitations. Child Development, 52, 1313-1317.

WeLLAN, H.M.; RITTER, K. y FLAVEIL, J.H. (1975). Deliberate memory behavior on the delayed reactions of very young children. Developmental Psychology, 11, 780-787.

WERTSCH. J.V. (1979). The regulation of human action and the given-new organization of private speech. En G. Zivin (Ed.): The development of self-regulation through private speech. Nueva York: Wiley.

ZINCHENKo, P.I. (1983-84). The problem of involuntary memory. Soviet Psychology, 22, 2, 55-111.

ZIVIN, G. (1979). Removing common confusions about egocentric speech, private speech and selfregulation. En G. ZIVIN (Ed.): The development of self-regulation through private speech. Nueva York, Wiley.

\section{Extended summary}

The goal of this paper is to present a theoretical schema of memory processes. This schema integrates contributions from different research lines.

- Research on levels of processing.

- Soviet investigation on memory and activity.

- Developmental studies on memory strategies and questions such as metamemory and memory control.

Despite their diverse origin and point of departure, they are all integrable in a single memory model because they share an active view of memory. The integration proposed is undertaken from a Vygotskian perspective, with the assumption that memory development is determined by social interactions. Speech is regarded as playing an essential role both in development and in memory processes functioning. After presenting the main issues of these research lines, the general schema for integrating their different contributions is proposed. 


\section{LEVELS OF PROCESSING}

Researchers in levels of processing framework argued that memory retention depended on stimuli analysis (semantic or shallow). They proposed the concept of depth of processing to take into account the differences in retention associated with qualitative differences in stimuli analysis. Later, this idea was completed by introducing concepts like:

- Elaboration: quantitative concept for considering analysis strength and number of encoded features.

- Distinctiveness: this notion emphasises the need to take into account encoding context and discriminative value of analysis.

All these concepts regard encoding processes. From a different but related point of view, Tulving formulated the notion of encoding specificity concerning retrieval processes (encoding and retrieval processes relationship).

\section{MEMORY ACTIONS}

In the previous paper we stated that Western research on memory strategies and Soviet investigation on memory and activity share and instrumental and goal-directed behaviour approach to memory. Strategies are memory deliberate actions. Recent research in Western psychology has shown that the development of strategies, as Soviet authors pointed out many years before, begin early in the infants life and go on for a long period to adulthood. At the same time, the individual's mastery of any strategy not only depends on his/her age, but on factors such as materials, problem solving situations and tasks must be considered as well.

\section{CONTROL PROCESSES}

Memory control processes include, as an important constitutive element, knowledge of factors affecting memory. The concept of metamemory refers to this kind of knowledge. Studies on metamemory development has focused on two main topics: awareness of the need to memorize and knowledge of different factors affecting one's memorization and recall. Terminological reasons led us to reserve the term metamemory only for knowledge factors, without including the control processes under its domain. Recent research on metamemory and memory control processes have put in evidence, as we pointed out in the former paper, the existence of the very long and complex development of them, from early infancy to adulthood.

\section{SPEECH AND MEMORY CONTROL}

Adopting a Vygotskian perspective on memory development implies the assumption of the mediational nature of memory and, therefore, the importance of speech as the very instrument of both acquisitiong and operation of memory strategies and control processes. As a genuine action system, speech make posible actions monitoring and change. It participates in memory actions (strate- 
gies), from very simple to more complex ones and in memory plans and control processes. All these processes, despite their different levels of complexity, possess a verbal nature. The role of verbal activity in the regulation of actions is determined by the predicative character of regulatory speech (social or «privatex), which omits given information preserving only new one (Wertsch, 1979) and directs the subject's attention to the most relevant aspects of the task and situation.

\section{CONCLUSION}

Once the main issoes in each line of research have been described, the general schema for integrating them can be presented, since they focus on different level processes and are likely to be coordinated into a single model. Concepts at the levels of processing framework refer to simple memory processes, that is, memory operations, in Soviet terminology. Memory strategies are situated at a different level. They are genuine actions which are more or less simple or complex. What Soviet and Western researchers have studied is the development of these actions, namely the determinants of the subject's acquisition and mastery of them. Finally, in the higher level of hierarchy we can place memory control processes. They are high-level strategies, elaborated actions (including subordinated ones) useful to carry out complex memory tasks. The inclusion of speech in this model has two main implications: as an action system, speech is the mediational instrument for the acquisition and working of memory actions and operations; as a social communicative system, its inclusion means recognizing the social character of memory development, an issue was analized in more detail in the first of these papers. 